\title{
Relationship of IGF-I and CKMB in Ischemic heart disease subjects
}

\begin{abstract}
Background: Ischemic heart disease (IHD) as one of cardiovascular disease becomes a major public health concern worldwide. IGF-1 is a prominent biomarker of IHD risk.

Objective: The aim of this study was to evaluate the relationship of IGF-1 with IHD and its potential risk factor in subjects visiting Punjab Institute of Cardiology, Lahore.

Methodology: For this purpose case-control study was done in Punjab Institute of Cardiology Lahore, Pakistan on 75 subjects. All the subjects were divided into two groups as IHD group $(n=50)$ and control group $(n=25)$. IHD subjects were taken from Punjab Institute of Cardiology Lahore, Pakistan, after the ethical approval of the committee. Control group subjects were enrolled from normal healthy population. Biochemical parameters such as lipid profile were assessed by using chemistry analyzer. ELISA was performed for the cardiac enzyme (CK-MB) and hormonal assessment (IGF-1) in the laboratory of LCWU. Statistical analysis was done by using SPSS version 20.0 (ILO, Chicago).
\end{abstract}

Results: High percentage of smoking $(60 \%)$, family history of cardiovascular diseases $(62 \%)$ and high percentage of physical inactivity activity $(70 \%)$ was found out in IHD group. Total Cholesterol, CK-MB and IGF-1 were significantly higher in IHD group as compared with the control group. The relationship of IGF-1 with TG and CK-MB was direct though non-significant.

Conclusion: It is concluded that IGF-1 is an important risk factor for the progression of IHD as IGF-1 was directly related with risk factors of IHD including triglycerides and CKMB while indirectly associated with total cholesterol.

Keywords: IHD, IGF-1, pakistani population, cholesterol, CKMB
Volume 6 Issue 5 - 2018

\author{
Saima Sharif,' Muhammad Saqib, ${ }^{2}$ Shagufta \\ Naz,' Misbah Manzoor,' Afshan Kaleem, ${ }^{3}$ \\ Mehwish lqtedar ${ }^{3}$ \\ 'Department of Zoology, Lahore College for Women University, \\ Pakistan \\ 2Department of Gastroenterology, Shaukat Khanum Hospital, \\ Pakistan \\ ${ }^{3}$ Department of Biotechnology, Lahore College for Women \\ University, Pakistan
}

\begin{abstract}
Correspondence: Saima Sharif, Department of Zoology, Lahore College for Women University, Lahore, Pakistan, Tel (042)99203801,Email ssharif1978@yahoo.com
\end{abstract}

Received: July 30, 2018 | Published: September 06, 2018

\section{Introduction}

Insulin-like growth factor-1 (IGF-1) is a protein that in humans is encoded by the IGF-1 gene. ${ }^{1}$ IGF-1 circulates in plasma, predominantly as the protein-bound form, while the free active peptide represents only a small percentage (less than $1 \%$ ) of the total circulation. ${ }^{2}$

IGF-1 plays an important role in childhood growth and continues to have anabolic effects in adults. IGF-1 production occurs throughout life. The highest rates of IGF-1 production occur during the pubertal growth spurt while the lowest levels occur in infancy as well as in old age. IGF-1 is among several factors that have been suggested to regulate pre degenerative abnormalities. ${ }^{3}$

IGF-1 can promote coronary arteriolar dilation through activation of potassium channels. ${ }^{4}$ It can induce the production of nitric oxide by vascular endothelium and substantially reduce intracellular calcium ${ }^{5}$ and infarct size in different models of myocardial ischemia ${ }^{6}$ IGF-1 either endogenous or exogenous is known to increase myocardial contractility in the short and long term. ${ }^{7}$ IGF-1 enhances insulin sensitivity, increases plasma glucose disappearance and tissue glucose utilization in healthy subjects. ${ }^{8}$

Coronary atherosclerosis is the major culprit of IHD. When the atherosclerotic plaques are stable, a frequent episode of chest pain occurs. However, when the breakdown of plaque occurs, myocardial infarction (MI) appears. ${ }^{9}$ CVD is not only the problem of the West $^{10}$ because $86 \%$ of the global burden of CVD is in the developing countries. Despite the high death rates due to non-communicable diseases, the leading cause of death, by 2010, in the developing countries including Pakistan would be CVD ${ }^{11}$.
In the developing countries the increase in ischemic heart disease (IHD) mortality (120\% in women and a 137\% in men), between 1990 and 2020, is expected to be much greater than among developed countries ( $29 \%$ and $48 \%$, respectively). ${ }^{12}$

The rise in IHD reflects a significant change in diet habits, physical activity levels, and tobacco consumption worldwide. High blood pressure, high blood cholesterol, overweight and obesity and the chronic disease of type 2 diabetes are among the major biological risk factors. ${ }^{11}$ Both low and high levels of circulating IGF1 have been reported to be associated with increased mortality and with cardiac failure. ${ }^{13}$

High concentrations of IGF-1 were associated with chronic heart failure and $50 \%$ greater risk for dying of cardiovascular causes. ${ }^{14}$ There was a tendency toward an association between high IGF-1 levels and all-cause mortality. So this study was performed to assess the levels of insulin like growth factor-1 in subject's suffering from ischemic heart disease.

\section{Materials and methods}

This case control study was conducted in Punjab Institute of Cardiology Lahore from January to July 2016. Data was collected from emergency department and from Jilani Block. In this study 75 subjects were sampled. Sample size was calculated by using sample calculator on Raosoft with 93\% confidence level, 9\% margin of error and taking expected respone distribution as $75 \%$.

The study population was divided into two groups, IHD group $(n=50)$ and control group $(n=25)$. Normal healthy individuals were 
taken as control group for comparison. IHD was diagnosed on the basis of electrocardiographic changes by the medical staff of PIC. Diabetic and hypertensive individuals were taken randomly. The study was approved by the Ethical Review Committee of Punjab Institute of Cardiology, Lahore.

The data was collected through a self-designed questionnaire. The questionnaire included information regarding each selected patient's demographic data including age (years), Sex, body mass index, socioeconomic status, and medical history including duration of IHD, family history of IHD, diabetes, and hypertension. Use of alcohol, smoking, type of food and physical activity were recorded.

Lipid profile was assessed by using chemistry analyzer and IGF-1 was assessed by using ELISA on automated ELISA system.

\section{Statistical analysis}

The datasheet was made on MS excel 2010. Data was expressed as mean \pm SEM. Difference between the groups was determined with the help of independent T-test. While all the statements of significance were based on the probability level of 0.05 and highly significant were based on 0.01 level of probability at $95 \%$ confidence interval.
The relationship between the parameters was found out by bivariate Pearson correlation analysis. The analysis was done with the help of SPSS version 20.0 (ILO, Chicago).

\section{Results}

For this study, data was collected from 75 subjects. Demographic data and biochemical parameters with their mean values were shown in Table 1. In IHD group 70\% were male with mean age of 62.54 years \pm 1.36 and $30 \%$ were female with mean age of 58.66 years \pm 2.03. IGF-1 levels were higher in IHD group $6.56 \pm 1.17 \mathrm{ng} / \mathrm{ml}$ and lower in control group $1.41 \pm 0.50$. High level IGF-I was significantly associated with increased risk of IHD.

Individuals with IHD had higher BMI, blood pressure, total cholesterol and triglycerides compared with controls (Table 1). Furthermore, smoking and use of alcohol were more prevalent among IHD group versus control group.

In order to further elucidate we correlate the relationship of IGF-1 with lipid profile and cardiac enzyme by two-tailed Pearson correlation analysis.

Table I Demographic and biochemical parameters of IHD and Control groups

\begin{tabular}{|c|c|c|c|c|c|c|}
\hline Variables & $\begin{array}{l}\text { Control group } \\
(n=25)\end{array}$ & & & $\begin{array}{l}\text { IHD group } \\
(n=50)\end{array}$ & & \\
\hline & All & Male & Female & All & Male & Female \\
\hline Age (Years) & $62.52 \pm 1.47$ & $63.11 \pm 1.77$ & $61.11 \pm 2.74$ & $61.36 \pm 1.14$ & $62.5 \pm 1.36$ & $58.66 \pm 2.03$ \\
\hline BMI $\left(\mathrm{Kg} / \mathrm{m}^{2}\right)$ & $24.22 \pm 0.66$ & $23.86 \pm 0.96$ & $25.36 \pm 0.54$ & $25.11 \pm 0.39$ & $23.82 \pm 0.4$ & $23.08 \pm 0.94$ \\
\hline $\begin{array}{l}\text { Systolic Blood } \\
\text { Pressure (mm } \\
\mathrm{Hg})\end{array}$ & $122.0 \pm 1.5^{*}$ & $121.7 \pm 2.14$ & $122.5 \pm 1.3$ & $129.9 \pm 2.54 *$ & $126.6 \pm 2.7$ & $137.6 \pm 5.3$ \\
\hline $\begin{array}{l}\text { Diastolic Blood } \\
\text { Pressure } \\
(\mathrm{mmHg})\end{array}$ & $85.44 \pm 2.40$ & $83.58 \pm 2.16$ & $89.3 \pm 5.03$ & $81.90 \pm 1.40$ & $81.9 \pm 1.4$ & $85.66 \pm 2.48$ \\
\hline TC (mg/dl) & $170.63 \pm 7.9 * *$ & $170.5 \pm 5.4^{a}$ & $170.9 \pm 10.8$ & $200.29 \pm 5.9 * *$ & $206.08 \pm 7.6^{a}$ & $\mid 87.11 \pm 7.9$ \\
\hline TG (mg/dl) & $148.7 \pm 13.7$ & $\begin{array}{l}132.8 \pm \\
12.29\end{array}$ & $182.32 \pm 32.5$ & $181.48 \pm 13.2$ & $194.4 \pm 17.6$ & $|5| . \mid \pm 13.8$ \\
\hline CK-MB (U/L) & $6.4 \pm 0.55$ & $6.17 \pm 0.6$ & $11.00 \pm 7.00$ & $83.4 \pm 6.9 * *$ & $80.7 \pm 7.7$ & $89.66 \pm 14.85$ \\
\hline $\begin{array}{l}\text { IGF-I } \\
(\mathrm{ng} / \mathrm{ml})\end{array}$ & $1.41 \pm 0.50$ & $0.92 \pm 0.29$ & $2.45 \pm 1.46$ & $6.56 \pm 1.17^{* *}$ & $6.09 \pm 1.37$ & $7.66 \pm 2.27$ \\
\hline
\end{tabular}

*: significant difference between the groups $(p \leq 0.05)$

**: highly significant difference between the groups $(p \leq 0.0 \mathrm{I})$

a: Highly significant differences between the males of control and IHD groups $(p \leq 0.0 I)$

There was a direct relation between IGF-1 and TC $(r=0.131)$ in control group. While in IHD group an indirect and non-significant correlation $(r=-0.038)$ was found. There was a direct and nonsignificant correlation between IGF-1 and TG in control group $(\mathrm{r}=0.056)$ as well as in IHD group $(\mathrm{r}=0.034)$.
In control group IGF-1 and CK-MB were inversely ( $\mathrm{r}=-0.195)$ correlated whereas direct $(\mathrm{r}=0.022)$ and non-significant correlation in in IHD group. This showed that amount of IGF-1 released in IHD subjects was positively associated with CK-MB release. The nonsignificant relationship might be due to small number of sample size. (Figure 1A) (Figure 1B) 


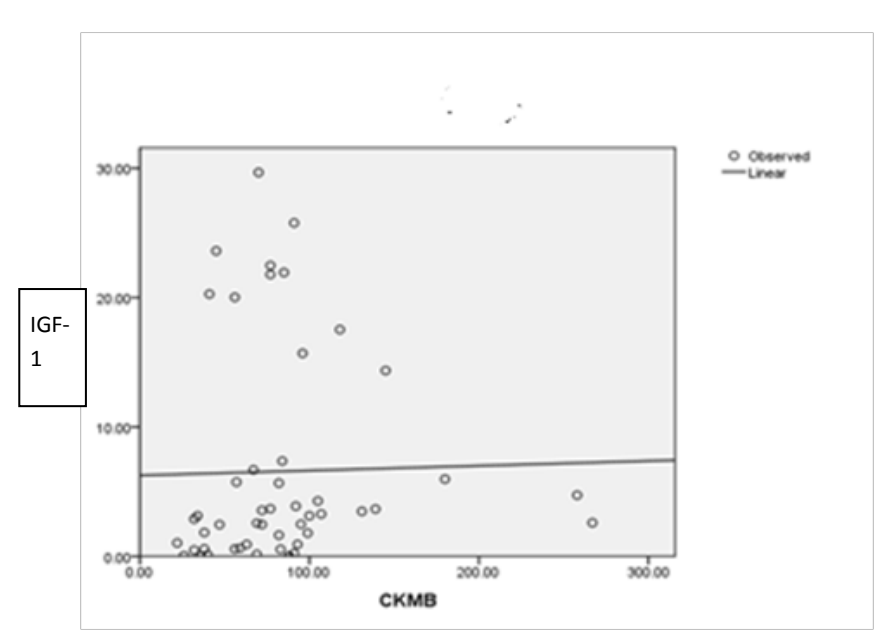

Figure IA Scattered plot showing Correlation between IGF-I (ng/ml)and CK-MB (U/L) in IHD group.

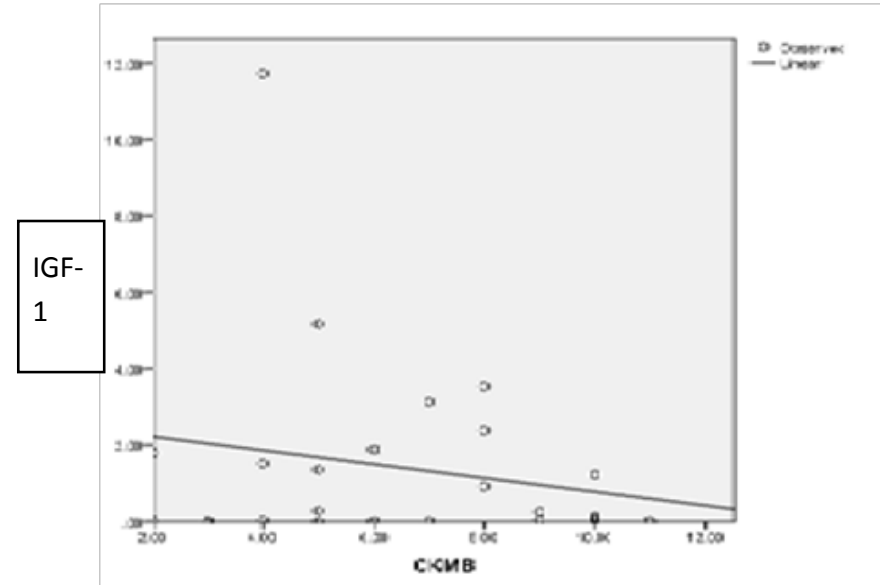

Figure IB Scattered plot showing Correlation between IGF-I (ng/ml) and CK-MB (U/L) in Control group.

\section{Discussion}

The developing countries contribute a greater share to the global burden of CVD than the developed countries15. In the year 2000 cardiovascular diseases (CVDs) accounted for 16.7 million deaths globally. ${ }^{12}$ South Asians are reported to have one of the highest rates of coronary heart disease. This trend has been seen in migrant South Asians as well as in those residing in their native countries. ${ }^{16}$ Pakistan, a developing country, has a population of over 130 million, a third of which is urban. According to the National Health Survey of Pakistan ${ }^{17,18}$ mortality due to CHD is on the rise.

Several studies have shown the difference in prevalence of IHD in gender specific manner. In present study the prevalence of IHD was higher in males (70\%) as compared to females (30\%). In present study the BMI was higher in IHD subjects than the control subjects. IHD subjects were overweight but not obese. Our findings showed that being overweight is a risk factor of Ischemic heart disease as described by Bogers 18 that overweight and obesity are associated with a significant increase in risk of CHD, and thus the worldwide increase in (moderate) overweight may drive the incidence of CHD upward.
In our study the mean values of blood pressure were higher in IHD group as compared to control group. In present study the mean of systolic blood pressure was significantly higher in IHD group as compared to control group while mean of diastolic blood pressure in IHD group was lower than the control group. In consistence to our study there was a trend of declining relative importance of diastolic and a corresponding increase in the importance of systolic pressure with advancing age. High blood pressure is a great risk factor of IHD which is also supported by other researcher. ${ }^{19}$

Total serum cholesterol is an independent risk factor of IHD. In our study the mean value of total cholesterol was high in IHD group and was lower in control group. Our results were supported by other studies. ${ }^{20}$ In present study the levels of serum triglycerides were higher in IHD group as compare to control group. There was non-significant relationship between the groups. Triglycerides have an association with risk of ischemic heart disease as reported by other studies..$^{21,22}$

CK-MB is a useful diagnostic biomarker for IHD and acute myocardial infarction. In present study the level of CK-MB was high in IHD subjects as compared to the control subjects. There was a highly significant difference between groups. In our study high level of CK-MB in IHD subjects indicated that there was myocardial damage to some extent, as blockage of coronary artery occurs in IHD which resulted into the release of CK-MB from cardiac monocytes. Our results were supported by other studies that there was increased level of CK-MB in IHD subjects. ${ }^{23}$

Level of IGF-1 is associated with risk of ischemic heart disease. In our study the level of IGF-1 was higher in IHD group and was lower in control group. So it was concluded that higher levels of IGF-1 causes ischemic heart disease and it was a strong prognostic factor for IHD. Other studies also supported our findings. ${ }^{24,25}$ It was reported 26 that persons with higher IGF1 levels had a $50 \%$ greater risk for dying of $\mathrm{CV}$ causes. Increased circulating levels of IGF-1 were characterized by a decreased incidence of heart failure and mortality in elderly individuals. ${ }^{27,28}$

In present study the relationship of IGF-1 and lipid profile was also determined. IGF-1 showed an indirect relationship with TC in IHD group but not in control group. The relationship was non-significant. Direct relationship of IGF-1 and TC was also reported ${ }^{29}$, in contrast to our study. There was a direct relationship between the IGF-1 and serum TG in IHD as well as in control group that indicated increase in IGF-1 also increases TG. Although the relationship was nonsignificant. It was supported by other studies. ${ }^{29}$

In the present study IGF-1 and CK-MB were directly related in IHD group but not in control group. This showed that amount of IGF1 released in IHD subjects was associated with CK-MB release.

\section{Conclusion}

It is concluded that IGF-1 is an important biomarker for the progression of IHD. Individuals with high circulating IGF-1 levels had increased risk of developing IHD, as there is direct relationship between IGF-I and CKMB.

\section{Acknowledgments}

The authors are thankful to the administration of Punjab Institute of Cardiology, Lahore for allowing sampling. 


\section{Conflict of interest}

Author declares that there is no conflict of interest.

\section{References}

1. Hoppener JW, Pagter-Holthuizen, P, Geurts KAH, et al. The human gene encoding insulin-like growth factor 1 is located on chromosome 12. Hum Genet. 1985;69(2):157-160.

2. Clemmons DR. Value of insulin-like growth factor system markers in the assessment of growth hormone status. Endocrinol Metab Clin North Am. 2007;36(1):109-120.

3. Torres I. Serum growth factors and neuroprotective surveillance: focus on IGF-1. Mol Neurobiol. 2000;21(3):153-160.

4. Izhar U. Masdai D. Richardson DM. Insulin and insulin-like growth factor-1 cause vasorelaxation in human vessels in vitro. Coron Artery Dis. 2000;11(1):69-76.

5. Inishi Y. Okuda T. Arakawa, T. et al. Insulin attenuates intracellular calcium responses and cell contraction caused by vasoactive agents. Kidney Int. 1994;45(5):1318-1325.

6. Kluge A, Zimmermann R, Weihrauch D. Coordinated expression of the insulin-like growth factor system after microembolisation in porcine heart. Cardiovasc Res. 1997;33(2):324-331.

7. Redaell G, Malhotra A, Li B. Effects of constitutive overexpression of insulin-like growth factor-1 on the mechanical characteristics and molecular properties of ventricular myocytes. Circ Res. 1998;82(5):594603.

8. Jones JI, Clemmons DR. Insulin-like growth factors and their binding proteins: biological actions. Endocr Rev. 1995;16(1):3-34.

9. Anke M, Smits FM, Smits M. Ischemic heart disease: models of myocardial hypertrophy and infarction. Cardio Di Dev. 2004;1(3):273278 .

10. Lopez AD. Global and regional burden of disease and risk factors, 2001: systematic analysis of population health data. Lancet. 2006;367(9524):1747-157.

11. Abbas S, Kitchlew AR, Abbas S. Disease Burden of Ischemic Heart Disease in Pakistan and its Risk Factors. Ann Pak Inst Med Sci. 2009;5(3):145-150.

12. Murray CJL, LopezAD. The Global Burden of Disease: A Comprehensive Assessment of Mortality and Disability from Diseases, Injuries, and Risk Factors in 1990 and Projected to 2020. 1996, Boston, Mass: Harvard School of Public Health.

13. Bunderen CC, Nieuwpoort IC, Schoor NM. The association of serum insulin-like growth factor-1 with mortality, cardiovascular disease, and cancer in the elderly; a population-based study. J Clin Endocri Metabo. 2010;95(10):4616-4624.

14. Chisalita SI. Dahlstrom U. Arnqvist HJ. et al. Increased IGF-1 levels in relation to heart failure and cardiovascular mortality in an elderly population: impact of ACE inhibitors. Eur J Endocri. 2011; 165(6):891898.
15. Whelton PK, Brancati FL. Appel LJ. The challenge of hypertension and atherosclerotic cardiovascular disease in economically developing countries. High Blood Pressure. 1995;4:36-45.

16. Bhopal R, Unwin N, White M, et al. Heterogeneity of coronary heart disease risk factors in Indian, Pakistani, Bangladeshi, and European origin populations: cross sectional study. Br Med J. 1999;319(7204):215-220.

17. National Health Survey of Pakistan (NHSP, 1998).

18. Bogers RP, Bemelmans WJ, Hoogenveen, RT, et al. Association of overweight with increased risk of coronary heart disease partly independent of blood pressure and cholesterol levels: a meta-analysis of 21 cohort studies including more than 300,000 persons. Arch Intern Med. 167(16):1720-1728.

19. Rosendorff C, Black HR, Cannon CP, et al. Treatment of hypertension in the prevention and management of ischemic heart disease: a scientific statement from the American Heart Association Council for High Blood Pressure Research and the Councils on Clinical Cardiology and Epidemiology and Prevention. Circulation. 2007; 29;115(21):2761-288.

20. Wilson PW, D-Agostino RB, Levy D, et al. Prediction of coronary heart disease using risk factor categories. Circulation. 1998;97(18):18371847.

21. Varbo A, Benn M, Tybjaerg-Hansen A, et al. TRIB1 and GCKR polymorphisms, lipid levels, and risk of ischemic heart disease in the general population. Thromb Vasc Biol. 2011;31(2):451-457.

22. Freiberg JJ, Tybjaerg-Hansen A, Jensen JS, et al. Nonfasting triglycerides and risk of ischemic stroke in the general population. JAMA. 2008;300(18):2142-2152.

23. Nallamothu BK, Bates ER. Periprocedural myocardial infarction and mortality. Causality versus association. $\mathrm{J} \mathrm{Am}$ Coll Cardiol. 2003;42(8):1412-1414.

24. Carlzon D, Svensson J, Petzold M, et al. Both low and high serum IGF-1 levels associate with increased risk of cardiovascular events in elderly men. J Clin Endocrinol Metab. 2014; 99(11):2308-2316.

25. Chisalita SI, Dahlstrom U, Arnqvist HJ, et al. Increased IGF-1 levels in relation to heart failure and cardiovascular mortality in an elderly population: impact of ACE inhibitors. Eur J Endocri. 2011;165(6):891898.

26. Laughlin GA, Barrett-Connor E, Criqui $\mathrm{MH}$, et al. The prospective association of serum insulin-like growth factor 1(IGF-1) and IGFbinding protein-1 levels with all cause and cardiovascular disease mortality in older adults: the Rancho Bernardo Study. J Clin Endocri Metabol. 2004;89(1):114-120.

27. Arcopinto M, Bobbio E, Bossone E, et al. The GH/IGF-1 axis in chronic heart failure. Endocr Metab Immune Disord Drug Targets. 2013;13(1):76-91.

28. Perkel D, Naghi J, Agarwal M, et al. The potential effects of IGF-1 and $\mathrm{GH}$ on patients with chronic heart failure. J Cardiovasc Pharmacol Ther. 2012;17(1):72-78.

29. Kawachi SI, Takeda N, Saski A, et al. Circulating Insulin-like Growth Factor Binding Protein-3 are associated with early carotid Atherosclerosis. Arterio Thromb Vasc Biol. 2005;25(3):617-621. 